\title{
IMPROVEMENT OF NETWORK LIFESPAN USING HANDOVER SCHEME IN WSN
}

\author{
Harsha.P.M ${ }^{1}$, Kanakaraju. $\mathbf{R}^{2}$ \\ ${ }^{1}$ Student, M.Tech (Digital Communication and Networking), TJIT, Bengaluru, Karnataka, India \\ ${ }^{2}$ Assistant Professor, Department of Electronics and Communication, TJIT, Bengaluru, Karnataka, India
}

\begin{abstract}
In the face of noteworthy improvements in wireless sensor networks (WSNs), energy management leftover is one of the most parts as an essential research challenges. Researchers have investigated architectures and topologies that allow energy proficient function of WSNs. One of trendy techniques in this gaze is at clustering. At same time as various research scholars encompassed investigate cluster head selection, this paper investigates formation of cluster. Particularly we put forward a novel schemes, LEACH-ERE along with Handover Mechanism is used for Enhancing the network lifetime. LEACH-ERE would be used in formation of cluster based on energy of nodes remained. Handover scheme used for competent cluster head selection and information transmission helps in maintaining existence for longer period.
\end{abstract}

Keywords: wireless sensor networks, handover, clustering, LEACH-ERE.

\section{INTRODUCTION}

Wireless Sensor Network consists of tiny sized, little cost sensor nodes, which be capable of sensing atmosphere and communicate the information gathered from monitored ground all way through wireless links. Information is forwarded by formation of clusters, just once clusters are formed; choose a cluster head which is used to combine information from distinct nodes in its cluster section. WSNs are information-centric, definite purpose, self-motivated nature, and scalable. Sensor node in WSN is pretended of four crucial components: sensing item, processing entity, transceiver element, and power block. Sometimes these nodes might have purpose reliant added workings [1].

Sensors are able to exchange information directly surrounded by them or to a sink deployed on the exterior in the neighbourhood. Although being independent, they boast inadequate battery, bandwidth and giving out power. Even among all resources constraints, majority concerning one is restricted energy [2]. One of foremost design goals of WSNs is to accomplish energy resourceful information communication although tiresome to make longer lifetime of network.

Design of device is classified as layered and clustered architecture. In clustering schemes, nodes form cluster within network, any one of a node would act as cluster head and other nodes perform as constituent node. Apparent information from a node is forwarded to $\mathrm{CH}$ which subsequently onward information to a node which is known as sink.
Layered architecture consists of the single and multiple hops to manage the data eventually where Base station collects all the data from the nodes for further processing while the clustered architecture consists of the nodes that are eventually distributed. Data from all the individual nodes are transferred to the cluster head which then forwards to the base station $\mathrm{CHs}$ farther away from the BS need to transmit data to a long distance in single-hop networks. Therefore, the energy of $\mathrm{CHs}$ farther away from the Base Station is larger than that of the $\mathrm{CHs}$ closer to the Base Station.

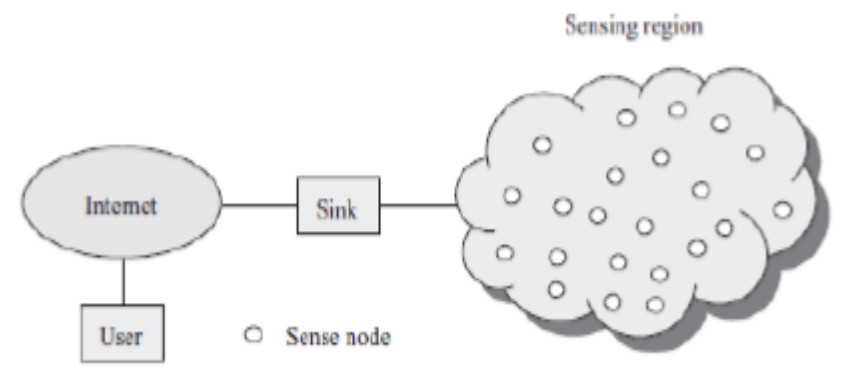

Fig-1: Architecture of WSN

Architectural design of WSN have high bang on energy utilization and thus network lifetime functioning. For the reason that energy limitation in nodes and only one of its kind many to one traffic model, remoteness information transfer is favoured in this kind of networks. Along with this, these networks involve a set of network protocols to carry out diverse control and supervision functions, which ought to capture keen on report not only on resource constraints, although purpose precise character of sensor network. 


\section{PROPOSED SYSTEM}

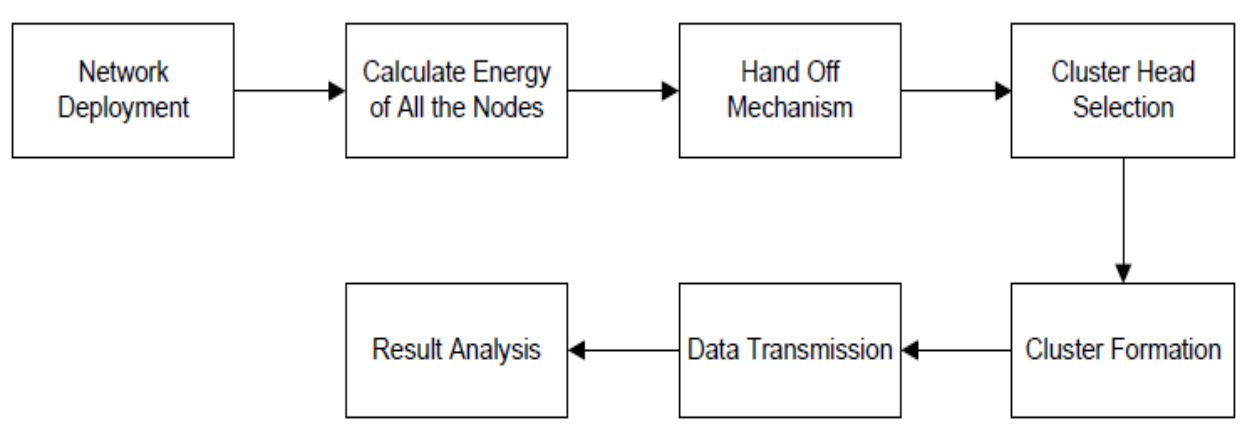

Fig-2: Block diagram of proposed System.

\subsection{Handover Procedure}

Handover [4] is a course of action through which nodes in movement obtain services and conserve traffic flows leading to happening a event link switch. Mechanisms and protocol layers concerned in this process can differ with variety of event link switching. Handover is necessitated as nodes would be moving in different directions without knowledge of neighbours. In order to have information transmission smoothly among nodes, they have to communicate with cluster head nodes in order to join with new clusters and send information messages to earlier cluster regarding put out of place of cluster.

Handover course of action occur in steps as follows:

Cluster detection - detection of neighbour cluster helps in computation of distance among nodes.

Cluster selection- Information exchange happens between nodes to join and send disjoin messages to prior clusters handover- Based on position and speed, a node is allowed to make handover process [5].

Handover efficiency always depends on level at which it is implemented and distinct sets of factors. On the other hand many of factors are of important in view of self-governing at which following method is performed. To begin with, availability of network information is vital in making decision of handover. In this paper, it will be based on received signal strength. Other forms grind over on several other parameters; all will be based at MAC level. Most of time location and speed will be parameters measured. Applications performing at terminals desires to be engaged into account, given that distinct applications have other requests.

\subsection{Static Based Leach-ERE Protocol}

Leach [11] stands for low energy adaptive hierarchical routing protocol which is organizing and adapting by itself. Since leach is a cluster centric, consisting of two levels: one is the set-up block and another is steady-state block. Steady state block is used for the intention of dipping the communication costs with the condition that the steady state block must be to a great extent higher than set-up block.

$$
T(n)= \begin{cases}\frac{p}{1-p \times\left(r \bmod \frac{1}{p}\right)} & n \in G \\ 0 & \text { others }\end{cases}
$$

During a method cluster arrangement will happen as node illogically selects a number from zero and one, compares this assessment with the subjected threshold values $\mathrm{T}$ (n) [7]. Then if the $T(n)$ is larger than specifies intermediate range we tend to take $T(n)$ because the cluster head. Values belonging to $T(n)$ is strong-willed by means of subsequently, wherever $\mathrm{p}$ be that the percentage of cluster heads altogether nodes, number of round is denoted by $r$; $G$ indicates nodes collection that haven't yet be head nodes within preliminary 1/P levels. Exploitation threshold value, every one node is going to be node head when 1/P levels are completed. Examination will proceed like this: every node has a chance to become cluster head with likelihood $\mathrm{p}$ when level starts, nodes that are head nodes during current level won't be node heads within next $1 / \mathrm{P}$ rounds, and as a result this number of nodes that is capable of becoming node head can reduce step by step. Thus remaining nodes will have chance of being head should be increased by $\mathrm{p}$. once 1/P-1 level is completed, all that haven't been head nodes are chosen head with likelihood 1, once 1/P levels are ended, Each nodes can come toward one and identical initial line.

\section{IMPLEMENTATION}

The procedure closest describes execution of proposed method, includes initialization which requires every node configuration contour energy, voltage, antenna kind etc. Next is pre initialization of nodes with labels to identify about information route and its nodes where arriving information is coming from. Subsequently, type of cluster supported their configuration and compute remaining energy of nodes. At this point foremost method in which cluster head of nodes is elected based in handover mechanism. Just once all these parameters are correct, there information exchange happens between members. 


\section{Initialize.}

Networks parameters initialization.

Set simulator objects, Topography objects.

Node configuration, position and labelling.

\section{Main Processing.}

Calculate distance between neighbouring node Detection of neighbouring clusters.

Calculate residual energy.

\section{Repeat}

Re-clustering formation.

Calculate distance between neighbouring node Detection of neighbouring clusters.

Calculate residual energy.

Cluster formation.

Cluster head selection.

Update routing path.

U'mill communication establishment.

Data transmission between nodes.

\section{Finalize.}

\section{Set NAM,}

Plot the $\mathrm{X}$-graph using trace files.

Fig-3: Procedure for proposed system.

\section{RESULTS AND DISCUSSIONS}

The figure 4 represents the graph over Energy Consumption vs. time for $M B$-LEACH-ERE. The graph clearly depicts the delivery ratio increases with respects to time and reaches saturation over a certain time.

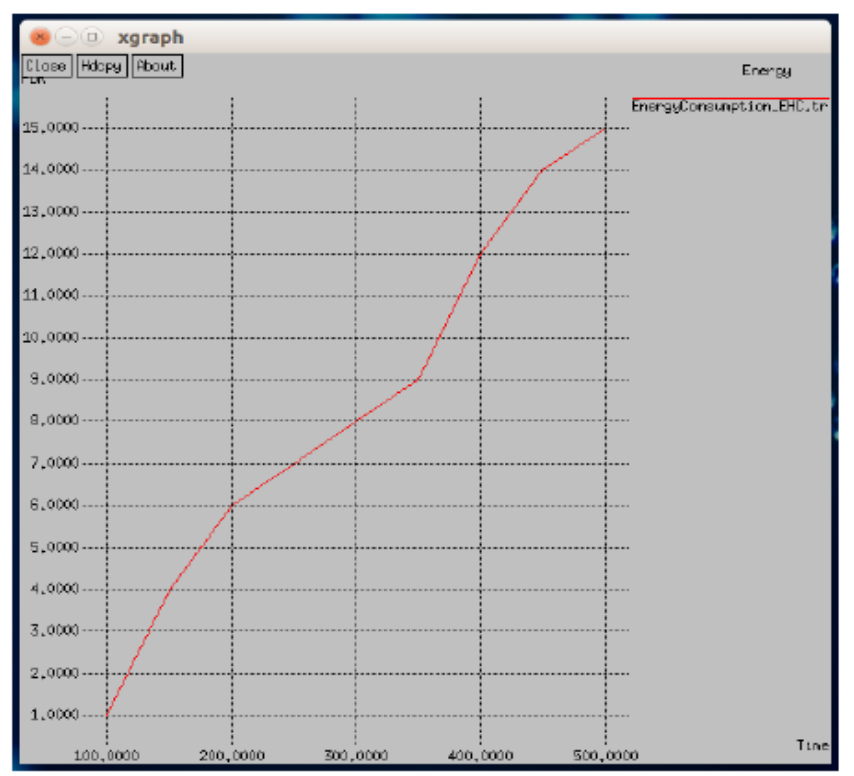

Fig-4: Energy Consumption vs. Time for $M B$-LEACH-ERE.
The figure 5 represents the graph over Packet delivery ratio vs. time for $M B$-LEACH-ERE. The graph clearly depicts the throughput increases with respects to time and reaches to the maximum over a certain time.

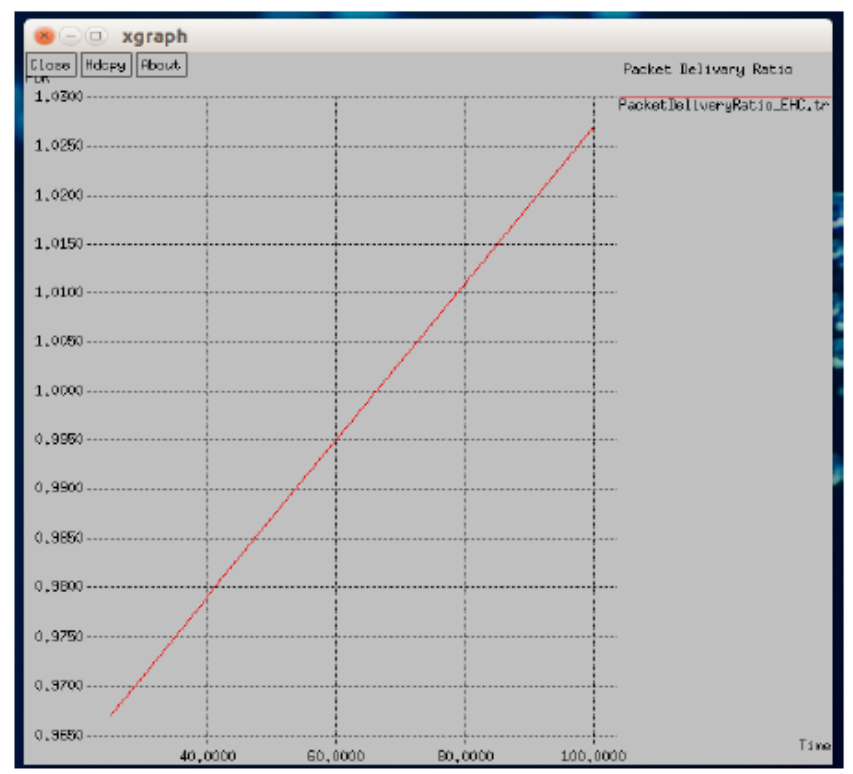

Fig-5: Packet delivery ratio vs. Time for $M B$-LEACH-ERE.

\section{CONCLUSION}

A novel and suitable approach has been approached to eliminate hands off and increase the throughput and packet delivery ratio using leach clustering and cluster head selection method using various low energy protocol with mobility in wireless sensor environment various technique to improve energy efficiency. Energy consumption and Packet delivery ratio get considerable fix in mobile nodes for each responsible node.

\section{REFERENCES}

[1]. C.Y. Chong and S. P. Kumar, "Sensor networks: Evolution, opportunities, and challenges", Proceedings of the IEEE, vol. 91 , no. 8, pp. 1247 - 1256, Aug. 2003.

[2]. Wireless LAN Medium Access Control (MAC) and Physical Layer (LHY) Specification, IEEE Std. 802.11 IEEE - 1999 edition.

[3]. Wong, K. D. and D. C. Cox, "A Pattern Recognition System for Handoff Algorithms", IEEE Journal on Selected Areas in Communication, Vol. 18, No. 7, July 2000.

[4]. S. Pack, et al., "Fast Handoff Support in IEEE 802.11 Wireless Networks," Communications Surveys \& Tutorials, IEEE, vol. 9, pp. 2-12, 2007.

[5]. W. Zhang, "Handover Decision Using Fuzzy MADM in Heterogeneous Networks," in Proc. of IEEE WCNC'04, Atlanta, GA, March 2004.

[6]. R.A.Roseline and Dr.P.Sumathi, Energy Efficient Routing Protocol and Algorithms for Wireless Sensor Networks-A Survey. Global Journal of Computer Science and Technology, vo1.11, December 2011.

[7]. J. Luo and J. - P. Hubaux, "Joint mobility and routing for lifetime elongation in wireless sensor networks", 
Proceedings IEEE INFOCOM' 05, vol. 3, Miami, FL, pp. 1735 - 1746, Mar. 2005.

[8]. W. Heinzelman, A. Chandrakasan, and $\mathrm{H}$. Balakrishnan , “An application - specific protocol architecture for wireless micro sensor networks ", IEEE Transactions on Wireless Communications, vol. 1, no. 4 , , pp. $660-670$, Oct. 2002.

[9]. V. Raghunathan, C. Schurgers, S. Park, and M. B. Srivastava, "Energy-aware wireless micro sensor networks”, IEEE Signal Proceedings Magazine, vol. 19, no. 2, pp. 40 - 50, Mar. 2002.

[10]. I. F. Akyildiz , W. Su , Y. Sankarasubramaniam , and E. Cayirci , "A survey on sensor networks ", IEEE Communications Magazine, vol. 40 , no. 8, pp. 102 - 114, Aug. 2002.

[11]. Jin-Shyan Lee, Senior Member, IEEE, and Wei-Liang Cheng, "Fuzzy-Logic-Based Clustering Approach for Wireless Sensor Networks Using energy predication" IEEE sensors journal, vol. 12, no. 9, September 2012.

\section{BIOGRAPHIES}

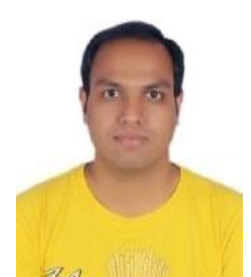

Mr. Harsha.P.M. is pursuing M.Tech degree in Digital communication \& Networking from T.John Institute of Technology, Bengaluru from Visvesvaraya Technological University. His research interests include Wireless Embedded Systems.

Communication, Operating Systems, and

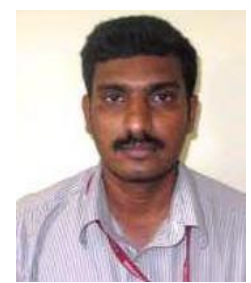

Mr Kanakaraju.R is an active researcher in the field of wireless sensor network, currently working as an Assistant Professor in Department of Electronics \& Communication at T.John Institute of Technology, Bengaluru. His research interest includes Digital

Communication and Communication Networks 